\title{
Understanding the transformation of spirals to lenticulars
}

\author{
Evelyn J. Johnston, Alfonso Aragón-Salamanca and \\ Michael R. Merrifield
}

School of Physics and Astronomy, University of Nottingham, University Park, Nottingham, NG7 2RD, UK

email: ejohnsto@eso.org

\begin{abstract}
By studying the individual star-formation histories of the bulges and discs of lenticular (S0) galaxies, it is possible to build up a sequence of events that leads to the cessation of star formation and the consequent transformation from the progenitor spiral. In order to separate the bulge and disc stellar populations, we spectroscopically decomposed long-slit spectra of Virgo Cluster S0s into bulge and disc components. Analysis of the decomposed spectra shows that the most recent star formation activity in these galaxies occurred within the bulge regions, having been fuelled by residual gas from the disc. These results point towards a scenario where the star formation in the discs of spiral galaxies are quenched, followed by a final episode of star formation in the central regions from the gas that has been funnelled inwards through the disc.
\end{abstract}

\section{Introduction}

Lenticular (S0) galaxies are often seen as an endpoint in the evolution of spiral galaxies since they display the same discy morphologies as spirals, but contain older stellar populations. In order to transform a spiral into an S0, the star-formation in the disc must be truncated and the bulge luminosity enhanced (Christlein \& Zabludoff 2004). Therefore, to understand how these two phenomena occur, we need to study the individual star-formation histories of the bulges and discs.

\section{Disentangling the Star Formation Histories of the Bulge and Disc}

A sample of 21 S0s from the Virgo Cluster were used in this study, with B-band magnitudes in the range of -17.3 to -22.3 and inclinations above 40 degrees to reduce contamination from misclassified ellipticals. A long-slit spectrum was obtained along the major axis of each galaxy using Gemini-GMOS with typical exposure times of $\sim 2-3$ hours. The reduced spectra were decomposed using the spectroscopic bulge-disc decomposition technique of Johnston et al. (2012), in which light profile of the galaxy in each wavelength bin was separated into bulge and disc components by fitting a Sérsic bulge plus exponential disc model. Having found the best fit and obtained the decomposition parameters for each wavelength bin, the total light from each component was then calculated by integration, and plotted against wavelength to create two one-dimensional spectra representing purely the bulge and disc light.

The ages, metallicities and $\mathrm{Mgb} /\langle\mathrm{Fe}\rangle$ ratios of the bulges and discs were measured using the strengths of the absorption features in the decomposed spectra. It can be seen in Fig. 1 that the bulges contain systematically younger and more metal rich stellar populations than their associated discs, suggesting that a final star-formation event occurred in the bulge region during the transformation. Additionally, a comparison of the $\mathrm{Mgb} /\langle\mathrm{Fe}\rangle$ 

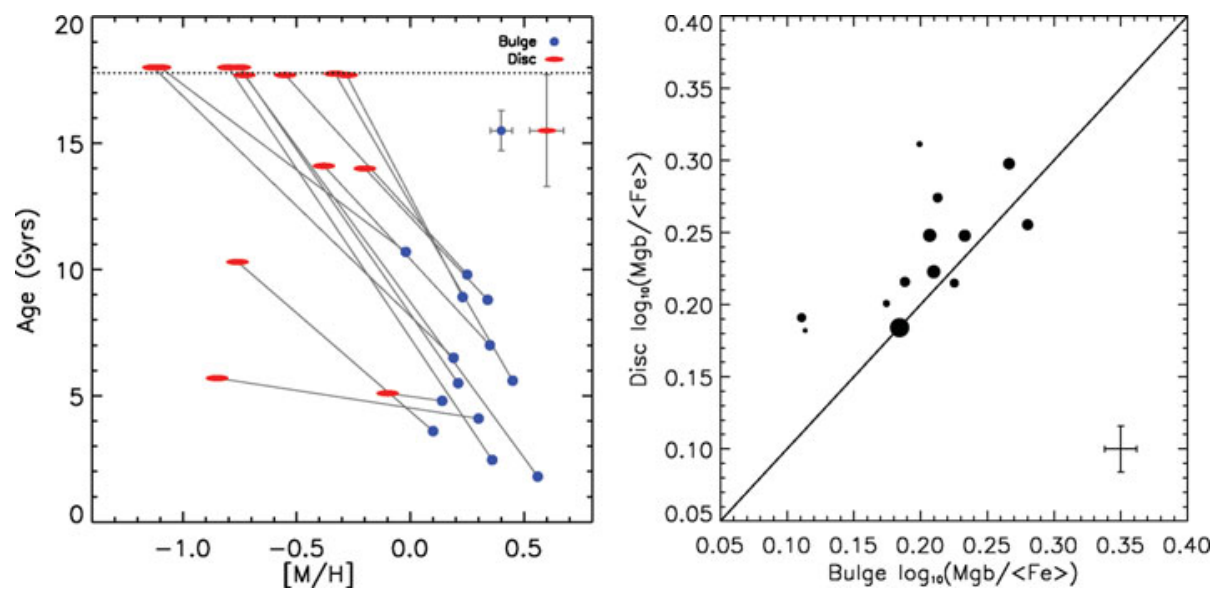

Figure 1. Left: The relative, light-weighted ages and metallicities of the bulges and discs of S0s in the Virgo Cluster. The lines link bulges and discs from the same galaxy. Right: Comparison of the $\mathrm{Mgb} /\langle\mathrm{Fe}\rangle$ ratios for the bulges and discs. Errors are shown on the right of each plot.

ratios for the bulges and discs, also shown in Fig. 1, reveals a correlation, indicating that their star-formation histories are linked.

\section{Conclusions}

We find that during the transformation from a spiral to an S0, gas is stripped from the disc gently enough that no significant amounts of star formation were triggered there. At some point, a final star-formation event occurs in the bulge region, resulting in younger, more metal rich stellar populations there. The most likely origin of the gas that fuelled this star formation is from the associated disc, which appears to be true from the clear correlation in the chemical enrichment of the the bulges and discs. Together, these results present a scenario for the transformation of spirals to S0s in which gas is stripped gently from the disc, while the residual gas is channelled in towards the centre of the galaxy. Eventually, this gas induces a final episode of star formation within the bulge, which uses up all the remaining gas and boosts the luminosity of the bulge relative to the disc. The whole galaxy then fades to an S0.

Clearly, further information on the transformation can be obtained if the decomposition was carried out in two dimensions using integral field unit (IFU) data. The up-coming IFU Mapping of Nearby Galaxies at APO (MaNGA, Bundy et al. in preparation) survey in SDSS-IV promises the resolution and wide field of view that will answer the remaining questions about how the bulge and disc star-formation histories are linked.

\section{Acknowledgements}

EJ acknowledges support from the STFC, the IAU and the RAS.

\section{References}

Christlein D. \& Zabludoff A. I., 2004, ApJ, 616, 192

Côté P. et al., 2004, ApJS , 153, 223

Johnston, E. J., Aragón-Salamanca, A., Merrifield, M. R., \& Bedregal, A. G. 2012, MNRAS, 422,2590 